\title{
Knowledge, Attitudes on Fall and Awareness of Hospitalized Patient's Fall Risk Factors Among the Nurses Working in Tertiary Care Hospitals.
}

\author{
Kavin Mozhi James \\ Panimalar College of Nursing \\ Divya Ravikumar \\ Panimalar Medical College Hospital \& Research Institute \\ Sindhura Myneni \\ Panimalar Medical College Hospital \& Research Institute \\ Poonguzhali Sivagnanam \\ Panimalar College of Nursing \\ Poongodi Chellapandian \\ Panimalar College of Nursing \\ Rejili Grace Joy Manickaraj \\ Panimlar College of Nursing

\section{Yuvasree Sargunan} \\ Panimalar Institute of Technology Department of Computer Science and Engineering \\ Sai Ravi Teja Kamineni \\ Panimalar Medical College Hospital \& Research Institute \\ Krishna Mohan Surapaneni ( $\square$ krishnamohan.surapaneni@gmail.com ) \\ Panimalar Medical College Hospital \& Research Institute https://orcid.org/0000-0002-5204-5708
}

\section{Research article}

Keywords: fall, fall risk factors, hospitalized patient and awareness

Posted Date: May 20th, 2020

DOI: https://doi.org/10.21203/rs.3.rs-28988/v1

License: @ (i) This work is licensed under a Creative Commons Attribution 4.0 International License. Read Full License 


\section{Abstract}

Back ground: Fall is the most common patient safety incident in health care organization. This study was initiated to obtain information regarding knowledge \& attitude on fall and awareness of fall risk factors among nurses to device evidence based and multidisciplinary educational and training programme to improve patient safety and thereby reducing morbidity and mortality associated with fall.

Methods: A descriptive cross sectional survey study was conducted among 339 registered nurses working in Tertiary care hospitals across Chennai, Tamil Nadu, India. Modified version of previously validated standard questionnaire was administered by the investigators through online survey method to explore the level of knowledge \&attitude on fall and awareness of inpatient fall risk factors among Nurses.

Results: In this study, $15.6 \%$ of participants had adequate knowledge on fall, $57.2 \%$ had favorable attitude towards fall and $38.3 \%$ adequate awareness on fall risk factors. Years of experience in nursing has statistical significant association with level of knowledge on fall. The participant's attitude towards fall had statistical significant relationship with age, education, experience in nursing and previous patient fall experience. The correlation between fall knowledge, attitude of fall and awareness of fall risk factors were highly significant. Majority of the participants expressed their favorable attitude towards need for fall preventive education.

Conclusion: In our study, it is evident that there is a void which has to be filled to improve the knowledge, attitude and awareness on fall and its risk factors. There is a need for extensive education and holistic, multifactorial and interdisciplinary training program to be undertaken through various health care organizations.

\section{Introduction}

A fall is defined as an event which results in a person coming to rest inadvertently on the ground or floor or other lower level (1). Falls and its consequences are major public health problem, making it the second leading cause of accidental deaths worldwide (2). Fall is reported as the most frequent patient safety event occurring in hospitals3. Fall occur at a rate of 3-5 per 1,000 occupied bed-days, and the Agency for Healthcare Research and Quality estimates that each year 700,000 to 1 million hospitalized patients fall (4). Nearly one in every three inpatients aged 65 years and older sustains fall. The traumatic complications of fall includes physical injury, functional impairment, increased rate of hospitalization and delayed recovery as a result people suffer a lot with a huge burden to the family, health sector, and economy of the country $(5,6,7)$. A significant proportion of non-traumatic falls, often instill fear of fall- a post fall syndrome which includes dependency, loss of autonomy, confusion, immobilization, and depression, which will lead to a further restriction in daily activities and thus affects Quality of life $(8,9)$. Despite the continued efforts to decrease falls in the hospital settings, there continues to be an increased incidence of inpatient falls. Nurses are the true backbone for any health facility. They provide constant care to patients and it is important that they are properly trained to identify risk factors and employ strategies to prevent them. Due to demanding working schedule, lack of awareness, complex patient profiles, there are high chances that preventable aspect of these "geriatric giants" are not well addressed and often overlooked. Knowledge and attitude of nurses attribute to disseminate and establish a foundation for fall preventive activities in Tertiary care hospitals. Even though falls among hospitalized patient is a well-recognized topic in both nursing and medical literature, in many developing countries it has not been prioritized for research. Hence, the current study had been devised to find out these lacunae and establish the best care for the patients.

\section{Materials And Methods}

\section{Study participants}

This is a cross sectional study was conducted in Panimalar Medical College Hospital \& Research Institute, Chennai, Tamil Nadu, India. A probability random sampling technique was used for selecting the study participants. The Researchers recruited 339 registered nurses who are working in tertiary care hospitals across Chennai aged over 20 years. Registered nurses who are able to read and write English and those willing to give informed consent were only included in the study. Nursing students, trainees and who were not available during data collection were excluded from participating in the study. A semi structured questionnaire was used to gather information from concerned participants at single point in time. Confidentiality of all participants was maintained by assigning unique respondent ID to each participant. This study protocol was approved by the Institutional Review Board (IRB) of the Panimalar Medical College Hospital \& Research Institute, Chennai (Panimalar Medical College Hospital \& Research Institute IRB \#1/2020/003) and conformed to the requirements of the Declaration of Helsinki (as revised in Seoul 2008).

\section{Measures}


Modified version of previously validated standard questionnaire was administered by the investigators comprised on 52 questions/ statements on demographic characteristics and information pertaining to the fall and its risk factors. The demographics were followed by 3 sections with set of questions/statements. The Section-1 incorporated 16 simple questions, to assess the knowledge on fall by selecting correct and incorrect options. Section - 2 embraced 13 positive and negative statements on 5 point likert scale, the respondents have to record their response on the 5 point Likert scale ranging from: Strongly agree (SA), Agree (A), Unsure (U), Disagree (D), Strongly Disagree (SD) for obtain information on attitude of fall and Sect. 3 - encompassed risk factor category question with the list of 16 risk factors where the respondent have to select related /unrelated option. The reliability of this tool was analyzed using Cronbach's alpha, wherein $a=0.75$.

\section{Statistical analysis}

Each statement/ question was numerically coded to obtain score for knowledge and attitude of fall and awareness of fall risk factors. All the categorical variables are presented as numbers and percentages. Descriptive analysis was performed using univariate statistics to report the Mean and Standard Deviation (SD) for the continuous variable and frequency distributions for the categorical variables. Correlation, non-parametric tests of Kruskal-Wallis and Mann-Whitney Test were performed to compare differences in the variables. All reported statistically significant differences were calculated at the $95 \%$ confidence level. Spearman's rho test measured the strength and direction of the relationship between two variables. Chi-square test was used to assess the association between categorical variables. Statistical significance was set at $p<0.05$. All statistical analyses were performed using Statistical Package for Social Science (SPSS, version 17) for Microsoft windows, SPSS Inc. USA.

\section{Results}

Of the 439 participants who were intended to receive survey questionnaires, 339 participants submitted completed questionnaires: a response rate of $75 \%$ leaving 339 valid for analysis. Table: 1 depicted the demographic characteristics of the nurses. Out of the participants $(n=339)$ are Female $(84.1 \%)$ and Male $(15.9 \%)$. Most of the nurses participated in the stud y $(36.3 \%)$ were their professional experience is less than 6 months. $21 \%$ of the respondent have experienced patients' that have sustained falls whereas (56.3\%) have not experienced previous patient fall. Majority of the respondent (71.7\%) have received fall prevention education. However, only (18.3\%) were extremely familiar with fall prevention activities.

\section{Knowledge on fall}

Considering overall level of knowledge of the 339 participants, 26.8\% ( $n=91)$ of individuals had inadequate knowledge, 57.5\% $(n=193)$ had moderately adequate knowledge, and $15.6 \%(n=53)$ had adequate knowledge on fall.(Fig. 1). The average mean knowledge of the study participants was $10.05 \pm 2.219$ (Table 5). Correlation between level of knowledge and participants' years of experience in nursing alone showed statistically significant relationship at p0.01 among all the selected demographic variable.(Table 6) Similarly only years of experience in nursing has statistical significant association (p0.01) with level of knowledge on fall.(Table 7)

The statements concerned with diseases condition and medications related to fall as expressed by the participants represented lowest of all knowledge scores were: $42 \%$ for "Taking medicine for diabetics is not related to fall" and "Intravenous therapy or intravenous access has a risk for fall,". $46 \%$ for "Depression is not related to fall and $47.2 \%$ for Taking medicines for blood pressure is not related to fall” (Table 2)

\section{Attitude towards fall}

In this present study, $2 \%(n=6)$ had unfavorable attitude, $42.2 \%(n=143)$ had moderately favorable attitude and $57.2 \%$ had favorable attitude towards fall.(Fig. 1) $49.69 \pm 5.746$ was the average mean attitude of the study participants.(Table 5) The participants' attitude towards fall had statistical significant relationship at p0.001 with age, education, experience in nursing and previous fall experience at p0.05. (Table 6)There were statistical significant associations between attitude of fall with age (0.05), gender (p0.001), educational qualification $(p<0.001)$ and experience in nursing $(p$ 0.001).(Table 7)

The lowest mean score for the statements on attitude of fall were: $2.58 \pm 1.24$ for the statement" hospital environment is safe for fall". $2.66 \pm 1.26$ for "fall is unavoidable" Majority of the participants expressed their favorable attitude towards need for fall preventive education. $4.56 \pm 0.74$ (Table.3)

\section{Awareness of fall risk factors}


The participants level of awareness on fall risk factors were 38.3\% $(n=130)$ adequate, $52.2 \%(n=177)$ moderately adequate and $9.4 \%(n$ = 32) inadequate.(Fig. 1) The average mean value was $11.48 \pm 2.165$. (Table 5) The correlation between fall knowledge, attitude of fall and awareness of fall risk factors were highly significant at p0.0001 with $95 \% \mathrm{Cl} .(T a b l e ~ 5)$ 
Demographic characteristics of the nurses included in the study ( $\mathrm{N}$ = 339)

\begin{tabular}{|c|c|c|}
\hline Demographics & Frequency & Percentage (\%) \\
\hline AGE & 47 & 13.9 \\
\hline$\leq 20$ years & 174 & 51.3 \\
\hline $21-25$ years & 56 & 16.5 \\
\hline $26-30$ years & 62 & 18.3 \\
\hline$>30$ years & 285 & 84.1 \\
\hline GENDER & 54 & 15.9 \\
\hline Female & 87 & 25.7 \\
\hline Male & 3 & .9 \\
\hline EDUCATIONAL QUALIFICATION & 205 & 60.5 \\
\hline Diploma in nursing \& & 44 & 13.0 \\
\hline Midwifery & 123 & 36.3 \\
\hline P.C.B.Sc & 75 & 22.1 \\
\hline B.Sc Nursing & 82 & 24.2 \\
\hline M.Sc Nursing & 19 & 5.6 \\
\hline EXPERIENCE IN NURSING & 40 & 11.8 \\
\hline Less than 6 months & 148 & 43.7 \\
\hline 6 months -2 years & 191 & 56.3 \\
\hline $2-6$ years & 243 & 71.7 \\
\hline $7-10$ years & 96 & 28.3 \\
\hline More than 10 years & 62 & 18.3 \\
\hline PREVIOUS PATIENT FALL & 103 & 30.4 \\
\hline EXPERIENCE & 126 & 37.2 \\
\hline Yes & 32 & 9.4 \\
\hline No & 16 & 4.7 \\
\hline \multicolumn{3}{|l|}{ RECEIVED FALL PREVENTION } \\
\hline \multicolumn{3}{|l|}{ EDUCATION } \\
\hline \multicolumn{3}{|l|}{ Yes } \\
\hline \multicolumn{3}{|l|}{ No } \\
\hline \multicolumn{3}{|l|}{ FALL PREVENTION ACTIVITY } \\
\hline \multicolumn{3}{|l|}{ Extremely familiar } \\
\hline \multicolumn{3}{|l|}{ Very familiar } \\
\hline \multicolumn{3}{|l|}{ Somewhat familiar } \\
\hline \multicolumn{3}{|l|}{ Not so familiar } \\
\hline Not at all familiar & & \\
\hline
\end{tabular}


Table 2

Statements that evaluate participants knowledge on fall in this study $(\mathrm{N}=339)$

\begin{tabular}{|lcc|}
\hline Items & Mean & Standard Deviation \\
\hline Recurrence rate is high among anyone who has already experienced a fall & .67 & .472 \\
\hline Falls occur most frequently among safety incidents in hospitals. & .58 & .495 \\
\hline Falls increase an elderly persons' death rate. & .77 & .420 \\
\hline Elderly hip fractures occur by falls & .89 & .312 \\
\hline Sliding is not falling & .55 & .499 \\
\hline The more medicine you take, the higher your fall risk. & .58 & .494 \\
\hline The more diseases you have, the higher your fall risK & .66 & .473 \\
\hline Depression is not related to falls. & .46 & .499 \\
\hline Someone who has a visual impairment has a higher risk for falls. & .91 & .280 \\
\hline Being numb in the limbs is not related to falls & .57 & .496 \\
\hline Dysuria is a risk factor for falls. & .70 & .458 \\
\hline Taking medicine for diabetes is not related to falls. & .42 & .495 \\
\hline Taking medicine for blood pressure is not related to falls & .47 & .500 \\
\hline Hearing impaired is not related to falls & .53 & .500 \\
\hline Falls occur more when getting up from and down on beds in hospitals. & .86 & .343 \\
\hline IV therapy or IV access has a risk for fall & .42 & .495 \\
\hline
\end{tabular}

Table 3

Statements that evaluate the attitude of falls among nurses enclosed in the study ( $N=339)$

\begin{tabular}{|llll|}
\hline Items & Mean & Std. Deviation \\
\hline I am concerned about patient falls. & 4.39 & .726 \\
\hline I think falls among patients is unavoidable & 2.66 & 1.268 \\
\hline I think nurses are responsible for patients' falls. & 3.75 & 1.235 \\
\hline Fall prevention is higher priority for intervention. & 4.35 & .794 \\
\hline I have concern about nursing interventions for fall prevention & 4.29 & .872 \\
\hline Fall prevention interventions should be conducted actively & 4.37 & .725 \\
\hline A patients' fall risk level should be inspected when hospitalised. & 4.32 & .900 \\
\hline Falls preventive education is necessary. & 4.56 & .741 \\
\hline Falls occur because of patients. & 2.59 & 1.082 \\
\hline I will help immediately if someone asks for help when they move. & 4.53 & .750 \\
\hline Physical injury is not severe even if a fall happens & 3.07 & 1.218 \\
\hline The hospital environment is safe for falls. & 2.58 & 1.241 \\
\hline I feel guilty if my patient falls. & 4.21 & .908 \\
\hline
\end{tabular}

Table-4: Frequency and Percentage of level of awareness on fall risk factors among the nurses $(\mathrm{N}=339)$. 
Table 5

Correlation between knowledge, attitudes on fall and awareness of fall risk factors among nurses $(\mathrm{N}=339)$

\begin{tabular}{|c|c|c|c|}
\hline RISK FACTOR & CATEGORY & $n$ & Percentage (\%) \\
\hline \multirow[t]{2}{*}{ Gender } & Related & 182 & 53.7 \\
\hline & Unrelated & 157 & 46.3 \\
\hline \multirow[t]{2}{*}{ Educational level } & Related & 138 & 40.7 \\
\hline & Unrelated & 201 & 59.3 \\
\hline \multirow[t]{2}{*}{ Hearing } & Related & 247 & 72.9 \\
\hline & Unrelated & 92 & 27.1 \\
\hline \multirow[t]{2}{*}{ Depression } & Related & 254 & 74.9 \\
\hline & Unrelated & 85 & 25.1 \\
\hline \multirow[t]{2}{*}{ Taking medicine } & Related & 290 & 85.5 \\
\hline & Unrelated & 49 & 14.5 \\
\hline \multirow[t]{2}{*}{ Urinary incontinence } & Related & 205 & 60.5 \\
\hline & Unrelated & 134 & 39.5 \\
\hline \multirow[t]{2}{*}{ Number of chronic disease } & Related & 258 & 76.1 \\
\hline & Unrelated & 81 & 23.9 \\
\hline \multirow[t]{2}{*}{ Cognitive impairment } & Related & 283 & 83.5 \\
\hline & Unrelated & 56 & 16.5 \\
\hline \multirow[t]{2}{*}{ Insomnia } & Related & 294 & 86.7 \\
\hline & Unrelated & 45 & 13.3 \\
\hline \multirow[t]{2}{*}{ Age } & Related & 302 & 89.1 \\
\hline & Unrelated & 37 & 10.9 \\
\hline \multirow[t]{2}{*}{ Use of assistive device } & Related & 268 & 79.1 \\
\hline & Unrelated & 71 & 20.9 \\
\hline \multirow[t]{2}{*}{ Anxiety for fall } & Related & 276 & 81.4 \\
\hline & Unrelated & 63 & 18.6 \\
\hline \multirow[t]{2}{*}{ Sight } & Related & 291 & 85.8 \\
\hline & Unrelated & 48 & 14.2 \\
\hline \multirow[t]{2}{*}{ Walking disorder } & Related & 313 & 92.3 \\
\hline & Unrelated & 26 & 7.7 \\
\hline \multirow[t]{2}{*}{ Activities of daily living } & Related & 262 & 77.3 \\
\hline & Unrelated & 77 & 22.7 \\
\hline \multirow[t]{2}{*}{ Dizziness } & Related & 306 & 90.3 \\
\hline & Unrelated & 33 & 9.7 \\
\hline
\end{tabular}


Table 6

Correlation of different characteristic variables with knowledge and attitude of fall

\begin{tabular}{|c|c|c|c|}
\hline Variables & Mean & S.D & Spearman's rho \& p-value, Sig. \\
\hline Knowledge & 10.05 & 2.22 & $r=0.206$ \\
\hline Attitude & 49.7 & 5.75 & $p=0.001$ \\
\hline Knowledge & 10.05 & 2.22 & $r=0.252$ \\
\hline Awareness on fall risk factors & 11.48 & 2.16 & $p=0.001$ \\
\hline Attitude & 49.7 & 5.75 & $r=0.214$ \\
\hline Awareness on fall risk factors & 11.48 & 2.16 & $p=0.001$ \\
\hline
\end{tabular}


Table 7

Association of demographic variables with level of knowledge and level of attitude among participants enclosed in this study.

\begin{tabular}{|c|c|c|c|c|c|c|c|}
\hline \multirow[t]{2}{*}{ Variables } & & \multicolumn{3}{|c|}{ Knowledge on Fall } & \multicolumn{3}{|c|}{ Attitude on Fall } \\
\hline & & $\mathbf{N}$ & $\begin{array}{l}\text { Mean } \\
\text { Rank }\end{array}$ & & $\mathbf{N}$ & $\begin{array}{l}\text { Mean } \\
\text { Rank }\end{array}$ & \\
\hline Kruskal-Wallis Test & Kruskal-Wallis Test & & & & & & \\
\hline \multirow[t]{4}{*}{ Age } & $\leq 20$ Years & 47 & 155.93 & $\begin{array}{l}\mathrm{H}= \\
7.010\end{array}$ & 47 & 143.17 & $\begin{array}{l}\mathrm{H}= \\
23.831\end{array}$ \\
\hline & $21-25$ Years & 174 & 161.02 & d. $f=3$ & 174 & 157.05 & d.f $=3$ \\
\hline & $26-30$ Years & 56 & 191.46 & $\begin{array}{l}\mathrm{p}= \\
0.072\end{array}$ & 56 & 175.93 & $p=0.001$ \\
\hline & > 30 Years & 62 & 186.48 & N.S & 62 & 221.31 & \\
\hline \multirow[t]{3}{*}{ Gender } & Female & 285 & 170.28 & $\begin{array}{l}Z= \\
0.124\end{array}$ & 285 & 174.42 & \multirow{3}{*}{$\begin{array}{l}Z=1.911 \\
p=0.056 \\
\text { N.S }\end{array}$} \\
\hline & Male & 54 & 168.50 & 0.901 & 54 & 146.67 & \\
\hline & & & & N.S & & & \\
\hline \multirow[t]{4}{*}{ Educational Qualification } & $\begin{array}{l}\text { Diploma in Nursing and } \\
\text { Midwifery }\end{array}$ & 87 & 169.11 & \multirow{3}{*}{$\begin{array}{l}H= \\
0.052 \\
\text { d.f }=3 \\
p= \\
0.997\end{array}$} & 87 & 125.41 & \multirow{4}{*}{$\begin{array}{l}H= \\
37.086 \\
\text { d.f }=3 \\
p=0.001\end{array}$} \\
\hline & P.C.B.Sc & 3 & 159.00 & & 3 & 174.33 & \\
\hline & B.Sc Nursing & 205 & 170.33 & & 205 & 175.27 & \\
\hline & M.Sc Nursing & 44 & 170.95 & N.S & 44 & 233.32 & \\
\hline \multirow[t]{5}{*}{ Experience in Nursing } & Less than 6 Months & 123 & 165.21 & \multirow{2}{*}{$\begin{array}{l}H= \\
7.113 \\
d f=4\end{array}$} & 123 & 153.56 & \multirow{5}{*}{$\begin{array}{l}H= \\
36.388 \\
d . f=4 \\
p=0.001\end{array}$} \\
\hline & 6 Months - 2 Years & 75 & 153.81 & & 75 & 163.08 & \\
\hline & $2-6$ Years & 82 & 175.11 & \multirow{2}{*}{$\begin{array}{l}p= \\
0.130\end{array}$} & 82 & 151.40 & \\
\hline & 7-10 Years & 19 & 212.21 & & 19 & 228.61 & \\
\hline & More than 10 Years & 40 & 184.56 & N.S & 40 & 243.83 & \\
\hline \multirow[t]{3}{*}{ Previous fall experience } & Yes & 148 & 170.50 & \multirow{2}{*}{$\begin{array}{l}Z= \\
0.083 \\
p= \\
0.934\end{array}$} & 148 & 152.44 & \multirow{3}{*}{$\begin{array}{l}Z=2.909 \\
p=0.004\end{array}$} \\
\hline & No & 191 & 169.62 & & 191 & 183.61 & \\
\hline & & & & N.S & & & \\
\hline \multirow[t]{6}{*}{$\begin{array}{l}\text { Received fall prevention } \\
\text { education }\end{array}$} & Yes & 243 & 176.62 & \multirow[t]{4}{*}{$\begin{array}{l}Z= \\
1.997\end{array}$} & 243 & 176.14 & \multirow{6}{*}{$\begin{array}{l}Z=1.839 \\
p=0.066 \\
\text { N.S }\end{array}$} \\
\hline & & & & & & & \\
\hline & & & & & & & \\
\hline & No & 96 & 153.26 & & 96 & 154.45 & \\
\hline & & & & $\begin{array}{l}p= \\
0.046\end{array}$ & & & \\
\hline & & & & $S^{*}$ & & & \\
\hline \multirow[t]{4}{*}{ Fall prevention activity } & Extremely familiar & 62 & 149.93 & \multirow{4}{*}{$\begin{array}{l}H= \\
7.091 \\
\\
d . f=4 \\
p= \\
0.131\end{array}$} & 62 & 162.38 & \multirow{3}{*}{$\begin{array}{l}H=6.042 \\
\text { d. } f=4 \\
p=0.196\end{array}$} \\
\hline & Very familiar & 103 & 183.88 & & 103 & 185.34 & \\
\hline & Somewhat familiar & 126 & 175.00 & & 126 & 170.72 & \\
\hline & & & & & & & N.S \\
\hline
\end{tabular}




\begin{tabular}{|c|c|c|c|c|c|}
\hline \multirow[t]{3}{*}{ Variables } & & \multicolumn{2}{|c|}{ Knowledge on Fall N.S } & \multicolumn{2}{|c|}{ Attitude on Fal } \\
\hline & & $\mathbf{N}$ & Mean & $\mathbf{N}$ & Mean \\
\hline & Not so familiar & 32 & Rabl84 & 32 & Ragl84 \\
\hline & Not at all familiar & 16 & 167.38 & 16 & 143.44 \\
\hline
\end{tabular}




\begin{tabular}{|c|c|c|c|c|c|c|c|c|c|}
\hline \multirow{3}{*}{ Demographics } & & \multicolumn{4}{|c|}{ Level of Knowledge on fall } & \multicolumn{4}{|c|}{ Level of Attitude on fall } \\
\hline & & \multirow[t]{2}{*}{ Inadequate } & \multirow[t]{2}{*}{$\begin{array}{l}\text { Moderately } \\
\text { Adequate }\end{array}$} & \multirow[t]{2}{*}{ Adequate } & \multirow{2}{*}{$\begin{array}{l}\text { Chi- } \\
\text { Square } \\
\text { Test, } \\
\text { P- } \\
\text { value } \\
\text { \& Sig. }\end{array}$} & \multirow[t]{2}{*}{ Unfavorable } & \multirow[t]{2}{*}{$\begin{array}{l}\text { Moderately } \\
\text { Favorable }\end{array}$} & \multirow[t]{2}{*}{ Favorable } & $\begin{array}{l}\text { Chi- } \\
\text { Square } \\
\text { Test, }\end{array}$ \\
\hline & & & & & & & & & $\begin{array}{l}\text { P- } \\
\text { value } \\
\& \text { Sig. }\end{array}$ \\
\hline \multirow[t]{8}{*}{ Age } & \multirow{4}{*}{$\begin{array}{l}\leq 20 \\
\text { Years } \\
21-25 \\
\text { Years }\end{array}$} & 15 & 26 & 6 & \multirow{4}{*}{$\begin{array}{l}x^{2}= \\
6.695 \\
\text { d.f }=6 \\
P= \\
0.350\end{array}$} & 0 & 25 & 22 & \multirow{8}{*}{$\begin{array}{l}\chi^{2}= \\
14.391 \\
\text { d.f }=6 \\
P= \\
0.026\end{array}$} \\
\hline & & $(31.9 \%)$ & $(55.3 \%)$ & $(12.8 \%)$ & & $(0.0 \%)$ & $(53.2 \%)$ & $(46.8 \%)$ & \\
\hline & & 50 & 103 & 21 & & 2 & 81 & 91 & \\
\hline & & $(28.7 \%)$ & & & & $(1.1 \%)$ & $(46.6 \%)$ & $(52.3 \%)$ & \\
\hline & \multirow{2}{*}{$\begin{array}{l}26-30 \\
\text { Years }\end{array}$} & 12 & 32 & 12 & \multirow[t]{4}{*}{ N.S } & 0 & 22 & 34 & \\
\hline & & $(21.4 \%)$ & $(57.1 \%)$ & & & $(0.0 \%)$ & $(39.3 \%)$ & $(60.7 \%)$ & \\
\hline & \multirow[t]{2}{*}{$>30$ Years } & 14 & \multirow[t]{2}{*}{$34(54.8 \%)$} & \multirow{2}{*}{$\begin{array}{l}14 \\
(22.6 \%)\end{array}$} & & 0 & 15 & 47 & \\
\hline & & $(22.6 \%)$ & & & & $(0.0 \%)$ & $(24.2 \%)$ & $(75.8 \%)$ & \\
\hline \multirow[t]{5}{*}{ Gender } & \multirow[t]{2}{*}{ Female } & 78 & \multirow{2}{*}{$\begin{array}{l}161 \\
(56.5 \%)\end{array}$} & \multirow{2}{*}{$\begin{array}{l}46 \\
(16.1 \%)\end{array}$} & \multirow{2}{*}{$\begin{array}{l}\chi^{2}= \\
0.807 \\
\text { d. } f=2\end{array}$} & 0 & 115 & $\begin{array}{l}170 \\
(596 \%)\end{array}$ & \multirow{5}{*}{$\begin{array}{l}\chi^{2}= \\
13.813 \\
\text { d.f }=2 \\
P= \\
0.001\end{array}$} \\
\hline & & $(27.4 \%)$ & & & & $(0.0 \%)$ & $(40.4 \%)$ & & \\
\hline & \multirow[t]{3}{*}{ Male } & 13 & 34 & 7 & $\mathrm{P}=$ & 2 & 28 & 24 & \\
\hline & & $(24.1 \%)$ & $(63.0 \%)$ & $(13.0 \%)$ & 0.000 & $(3.7 \%)$ & $(51.9 \%)$ & $(44.4 \%)$ & \\
\hline & & & & & N.S & & & & \\
\hline Educational & Diploma in & 19 & 56 & 12 & $x^{2}=$ & 1 & 57 & 29 & $x^{2}=$ \\
\hline & and & $(21.8 \%)$ & $(64.4 \%)$ & & 3.319 & $(1.1 \%)$ & $(65.5 \%)$ & (33.3\%) & 33.282 \\
\hline & IVIuvoriery & & & & 6 & & & & d.f = 6 \\
\hline & P.C.B.Sc & 1 & 2 & 0 & $\mathrm{P}=$ & 0 & 1 & 2 & $P=$ \\
\hline & & $(33.3 \%)$ & $(66.7 \%)$ & $(0.0 \%)$ & 68 & $(0.0 \%)$ & (33.3\%) & $(66.7 \%)$ & \\
\hline & $\begin{array}{l}\text { B.Sc } \\
\text { Nursing }\end{array}$ & 60 & 111 & 34 & N.U & 1 & 77 & 127 & \\
\hline & & $(29.3 \%)$ & & & & $(0.5 \%)$ & $(37.6 \%)$ & & \\
\hline & M.Sc & 11 & 26 & 7 & & 0 & 8 & 36 & \\
\hline & veroming & $(25.0 \%)$ & $(59.1 \%)$ & (15.9\%) & & $(0.0 \%)$ & $(18.2 \%)$ & (81.8\%) & \\
\hline Experience & Less than & 31 & 78 & 14 & $x^{2}=$ & 0 & 61 & 62 & $x^{2}=$ \\
\hline & & $(25.0 \%)$ & $(63.4 \%)$ & & 20.814 & $(0.0 \%)$ & $(49.6 \%)$ & $(50.4 \%)$ & 488 \\
\hline & 6 Months & 21 & 47 & 7 & & 2 & 30 & 43 & 0 \\
\hline & & $28.0 \%)$ & $(62.7 \%)$ & $(9.3 \%)$ & $\begin{array}{l}P= \\
0.008\end{array}$ & $(2.7 \%)$ & $(40.0 \%)$ & $(57.3 \%)$ & $\begin{array}{l}P= \\
0.001\end{array}$ \\
\hline & $2-6$ Years & 28 & 35 & 19 & $S^{\star *}$ & 0 & 43 & 39 & \\
\hline & & $(34.1 \%)$ & $(42.7 \%)$ & & & $(0.0 \%)$ & $(52.4 \%)$ & $(47.6 \%)$ & \\
\hline & $7-10$ & 4 & 8 & 7 & & 0 & 4 & 15 & \\
\hline & & $(21.1 \%)$ & $(42.1 \%)$ & $(36.8 \%)$ & & $(0.0 \%)$ & $(21.1 \%)$ & $(78.9 \%)$ & \\
\hline & More than & 7 & 27 & 6 & & 0 & 5 & 35 & \\
\hline & & $(17.5 \%)$ & $(67.5 \%)$ & $(15.0 \%)$ & & $(0.0 \%)$ & $(12.5 \%)$ & $(87.5 \%)$ & \\
\hline $\begin{array}{l}\text { Previous } \\
\text { patient fall }\end{array}$ & Yes & 38 & 88 & $\begin{array}{l}221 \\
(14.9 \%)\end{array}$ & $x^{2}=$ & 1 & 73 & 74 & $x^{2}=$ \\
\hline experience & & (25.7\%) & (59.5\%) & & & $(0.7 \%)$ & (49.3\%) & (50.0\%) & \\
\hline
\end{tabular}




\begin{tabular}{|c|c|c|c|c|c|c|c|c|c|}
\hline \multirow{3}{*}{ Demographics } & & \multicolumn{4}{|c|}{ Level of Knowledge on fall } & \multicolumn{4}{|c|}{ Level of Attitude on fall } \\
\hline & & \multirow[t]{2}{*}{ Inadequate } & \multirow[t]{2}{*}{$\begin{array}{l}\text { Moderately } \\
\text { Adequate }\end{array}$} & \multirow[t]{2}{*}{ Adequate } & $\begin{array}{l}\text { Chi- } \\
\text { Square } \\
\text { Test, }\end{array}$ & \multirow[t]{2}{*}{ Unfavorable } & \multirow[t]{2}{*}{$\begin{array}{l}\text { Moderately } \\
\text { Favorable }\end{array}$} & \multirow[t]{2}{*}{ Favorable } & $\begin{array}{l}\text { Chi- } \\
\text { Square } \\
\text { Test, }\end{array}$ \\
\hline & & & & & $\begin{array}{l}\text { P- } \\
\text { value } \\
\& \text { Sig. }\end{array}$ & & & & $\begin{array}{l}\text { P- } \\
\text { value } \\
\& \text { Sig. }\end{array}$ \\
\hline & \multirow[t]{3}{*}{ No } & 53 & \multirow{3}{*}{$\begin{array}{l}107 \\
(56.0 \%)\end{array}$} & \multirow{3}{*}{$\begin{array}{l}31 \\
(16.2 \%)\end{array}$} & d.f $=2$ & \multirow{3}{*}{$\begin{array}{l}1 \\
(0.5 \%)\end{array}$} & \multirow{3}{*}{$\begin{array}{l}70 \\
(36.6 \%)\end{array}$} & \multirow{3}{*}{$\begin{array}{l}120 \\
(62.8 \%)\end{array}$} & d.f $=2$ \\
\hline & & $(27.7 \%)$ & & & $\begin{array}{l}P= \\
0.817\end{array}$ & & & & $\begin{array}{l}P= \\
0.061\end{array}$ \\
\hline & & & & & N.S & & & & N.S \\
\hline \multirow{5}{*}{$\begin{array}{l}\text { Received fall } \\
\text { prevention } \\
\text { education }\end{array}$} & \multirow[t]{2}{*}{ Yes } & 63 & \multirow{2}{*}{$\begin{array}{l}136 \\
(56.0 \%)\end{array}$} & \multirow{2}{*}{$\begin{array}{l}44 \\
(18.1 \%)\end{array}$} & \multirow{2}{*}{$\begin{array}{l}x^{2}= \\
3.986\end{array}$} & 1 & 97 & 145 & \multirow{5}{*}{$\begin{array}{l}\chi^{2}= \\
2.402 \\
\text { d.f }=2 \\
P= \\
0.301 \\
\text { N.S }\end{array}$} \\
\hline & & $(25.9 \%)$ & & & & $(0.4 \%)$ & $(39.9 \%)$ & & \\
\hline & \multirow[t]{3}{*}{ No } & 28 & 59 & 9 & \multirow{2}{*}{$\begin{array}{l}P= \\
0.136\end{array}$} & 1 & 46 & 49 & \\
\hline & & \multirow[t]{2}{*}{$(29.2 \%)$} & \multirow[t]{2}{*}{$(61.5 \%)$} & \multirow[t]{2}{*}{ (9.4\%) } & & \multirow[t]{2}{*}{$(1.0 \%)$} & \multirow[t]{2}{*}{$(47.9 \%)$} & \multirow[t]{2}{*}{$(51.0 \%)$} & \\
\hline & & & & & N.S & & & & \\
\hline \multirow{10}{*}{$\begin{array}{l}\text { Fall } \\
\text { prevention } \\
\text { activity }\end{array}$} & \multirow{2}{*}{$\begin{array}{l}\text { Extremely } \\
\text { familiar }\end{array}$} & 18 & 38 & 6 & \multirow{4}{*}{$\begin{array}{l}\chi^{2}= \\
15.086 \\
\text { d.f }=8 \\
P= \\
0.057\end{array}$} & 0 & 31 & 31 & $x^{2}=$ \\
\hline & & $(29.0 \%)$ & $(61.3 \%)$ & $(9.7 \%)$ & & $(0.0 \%)$ & $(50.0 \%)$ & $(50.0 \%)$ & 11.478 \\
\hline & \multirow{2}{*}{$\begin{array}{l}\text { Very } \\
\text { familiar }\end{array}$} & 27 & 55 & \multirow{2}{*}{$\begin{array}{l}21 \\
(20.4 \%)\end{array}$} & & 0 & 35 & 68 & \\
\hline & & $(26.2 \%)$ & $(53.4 \%)$ & & & $(0.0 \%)$ & $(34.0 \%)$ & $(66.0 \%)$ & $\begin{array}{l}P= \\
0.176\end{array}$ \\
\hline & Somewhat & 31 & 71 & 24 & N.S & 1 & 52 & 73 & N.S \\
\hline & & $(24.6 \%)$ & (56.3\%) & & & $(0.8 \%)$ & $(41.3 \%)$ & (57.9\%) & \\
\hline & Not so & 13 & 17 & 2 & & 1 & 17 & 14 & \\
\hline & & $(40.6 \%)$ & $(53.1 \%)$ & $(6.3 \%)$ & & $(3.1 \%)$ & (53.1\%) & $(43.8 \%)$ & \\
\hline & Not at all & 2 & 14 & 0 & & 0 & 8 & 8 & \\
\hline & & $(12.5 \%)$ & $(87.5 \%)$ & $(0.0 \%)$ & & $(0.0 \%)$ & $(50.0 \%)$ & $(50.0 \%)$ & \\
\hline
\end{tabular}

\section{Discussion}

Fall in hospitalized patients is almost always preventable $(10,11)$. Nurses with adequate knowledge and awareness on fall and its risk factors, are anticipated to bring about be a significant reduction in the prevalence of fall occurrence and positive influence on fall among hospitalized patients (12). In our present study, $43.7 \%$ of nurses had previous patient fall experience which is similar to that reported by various studies $(13,14)$. This is much higher than that reported by Kim et al $(15)$. These differences may be due to nature of hospital infrastructure, years of nursing training program and difference in patient profiles.

Despite the fact that majority of the participants (71.7\%) reported "received fall prevention education", only $15.6 \%$ had adequate level of knowledge which was lower when compared with the study reported by Kim et al (15), where $85.7 \%$ of nurses had high level of knowledge. Similar to our study, Laing et al (16) reported a lower level of knowledge among health workers about fall. In our study majority of the nurses had working experience of less than 2 years hence low level of knowledge regarding a complex multi factorial event like fall can be attributed.

In the assessment of knowledge session of the questionnaire statements pertaining to diseases condition, treatment related and medications related to fall as expressed by the participants represented lowest of all score similar with a previous studies $(15,16)$. This could be attributed to not incorporating multidisciplinary evidenced based training on fall with new perspective. In India, it is a harsh reality that many hospitals neither have standard policies and procedures nor training programs on falls and its prevention. Laing et al 
(16) revealed that knowledge and skills of nurses related to fall and its prevention was improved after the training confirmed the need for evidence based training. Hence, introduction of a care bundle approach frequent in-services and focus groups may enhance nurses learning and improve clinical outcomes.

This study revealed majority $57.2 \%$ had favorable attitude towards fall which supports the study by Kim CG et al (14) where the attitude level of nurses was $77.2 \%$ positive on fall. This may be due to the social and cultural empathy incorporated in training. Contradictory results have been shown in various other studies $(13,14,17)$. This may be due to difference in nature of study participants (students, workers).. However, the potential gaps in awareness of fall risk factors may be addressed through developing nurse sensitive, contextspecific fall-prevention clinical guidelines for practice in India.

In our study $52.2 \%$ had moderately adequate awareness on fall risk factors . In contrast $60 \%$ of health care professionals including nurses had little or some awareness on fall risk factors in a study $(18,19)$. Therefore, an onsite training program on awareness of fall risk factors designed for nurses is crucial and need of the hour in order to help them identify the most important risk factors for falls and translate the knowledge into practice. Majority of the participants (66.1\%) expressed need for fall preventive education in this study this achieved through developing multifactorial, multidisciplinary fall preventive educational program and introducing bundle approach on fall.

The years of experience in nursing showed both strong association and positive relationship with level of knowledge. This implies that the knowledge improves as they gain more working experience because they obtain more information and hands on experience. The attitude of nurses towards fall had strong associations with age, gender, educational qualification and experience in nursing. This implies that as their experience in nursing care increases with education and patient care the more the positive attitude they develop on fall and it preventive measure.

Interestingly, our study also showed a strong correlation between knowledge, attitude and awareness of fall preventive measures with each other. This is supported by several studies as well $(14,15,16,20)$. This shows that continued medical education and training of nurses is required to bring about an overall change in fall incidents in hospitalized patients.

\section{Conclusion}

In our study, it is evident that there is a void which has to be filled to improve the knowledge, attitude and awareness on fall and its risk factors. There is a need for extensive education and holistic, multifactorial and interdisciplinary training program to be undertaken through various health care organization including governmental and non-governmental organizations regarding fall and risk factors of fall to reduce fall occurrence thereby ensuring patient safety.

\section{Declarations}

Ethics approval and consent to participate: Institutional Review Board (IRB) Approval has been obtained prior to start of the study (Panimalar Medical College Hospital \& Research Institute IRB \#1/2020/003). Informed Consent has been obtained from all the participants of the study.

-Consent for publication: All authors provided their consent for publication of this manuscript.

-Availability of data and material: The data used to support the findings of this study are available from the corresponding author upon request.

-Competing interests: "All the authors declare no conflict of interest."

-Funding: "This research received no external funding".

-Authors' contributions: KMJ, DR, SM, SKM: having contributed equally to the work; PS, YS: contributed to data curation, validation, visualization and writing the manuscript; PC, RGJM, SRTK: contributed to formal analysis, validation, visualization and writing the manuscript; KMJ, DR, SM, PS, PC, RGJM, YS, SRTK \& SKM: All authors reviewed the manuscript and approved the submitted manuscript.

\section{-Acknowledgements:}

We are thankful to Dr. S. Porchelvan, MSc, MBA, PGDCA, PhD, Professor in Biostatistics for assisting with the statistical analyses. 
Mrs. Kavin Mozhi James is Professor in Department of Medical Surgical Nursing, Panimalar College of Nursing, Varadharajapuram, Poonamallee, Chennai - 600 123, Tamil Nadu, INDIA. E-mail: kavin1608@gmail.com

Dr. Divya Ravikumar is a Junior Resident in Department of Obstetrics \& Gynecology, Panimalar Medical College Hospital \& Research Institute, Varadharajapuram, Poonamallee, Chennai - 600 123, Tamil Nadu, INDIA. E-mail: divya.ravi.chennai@gmail.com

Dr. Sindhura Myneni is Senior Resident in Department of Obstetrics \& Gynecology, Panimalar Medical College Hospital \& Research Institute, Varadharajapuram, Poonamallee, Chennai - 600 123, Tamil Nadu, INDIA. E-mail: sindhuramyneni15@gmail.com

Mrs. Poonguzhali Sivagnanam is Principal cum Professor in Department of Medical Surgical Nursing, Panimalar College of Nursing, Varadharajapuram, Poonamallee, Chennai - 600 123, Tamil Nadu, INDIA. E-mail: kuzhaliram@gmail.com

Mrs. Poongodi Chellapandian is an Associate Professor in Department of Obstetrics \& Gynaecological Nursing, Panimalar College of Nursing, Varadharajapuram, Poonamallee, Chennai - 600 123, Tamil Nadu, INDIA. E-mail: cpoongodi@hotmail.com

Mrs. Rejili Grace Joy Manickaraj is Assistant Professor in Department of Mental Health Nursing, Panimalar College of Nursing, Varadharajapuram, Poonamallee, Chennai - 600 123, Tamil Nadu, INDIA. E-mail: rgracejoy_@yahoo.co.in

Ms. Yuvasree Sargunan is a Assistant Professor in Department of Computer Sciences, Panimalar Institute of Technology, Varadharajapuram, Poonamallee, Chennai - 600 123, Tamil Nadu, INDIA. E-mail: sargunanyuvasree@gmail.com

Dr. Sai Ravi Teja Kamineni is a Junior Resident in Department of Tuberculosis \& Respiratory Diseases, Panimalar Medical College Hospital \& Research Institute, Varadharajapuram, Poonamallee, Chennai - 600 123, Tamil Nadu, INDIA. E-mail: ravi.kaminenin@gmail.com

Dr. Surapaneni Krishna Mohan is Professor in Department of Biochemistry; Head of Department of Clinical Skills \& Simulation, Panimalar Medical College Hospital \& Research Institute, Varadharajapuram, Poonamallee, Chennai - 600 123, Tamil Nadu, INDIA. E-mail: krishnamohan.surapaneni@gmail.com

\section{References}

1. Lamb SE, Jørstad-Stein EC, Hauer K, Becker C, Prevention of Falls Network Europe and Outcomes Consensus Group. Development of a common outcome data set for fall injury prevention trials: the Prevention of Falls Network Europe consensus. J Am Geriatr Soc. 2005 Sep;53(9):1618-22.

2. World Health Organization. The global burden of disease: 2004 update. World Health Organization; 2008.

3. Healey F, Darowski A. Older patients and falls in hospital. Clinical Risk. 2012 Sep;18(5):170-6.

4. Healey FM. The prevention of patient falls in healthcare settings, with particular emphasis on the effect of bedrail use on falls and injury as part of multi-faceted interventions (Doctoral dissertation, Newcastle University).

5. Kumar A, Srivastava DK, Verma A, Kumar S, Singh NP, Kaushik A. The problems of fall, risk factors and their management among geriatric population in India. Indian Journal of community health. 2013 Jun 30;25(2):89-94.

6. Dsouza SA, Rajashekar B, Dsouza HS, Kumar KB. Falls in Indian older adults: a barrier to active ageing. Asian J Gerontol Geriatr. 2014 Jun;9(1):1-8.

7. Williams JS, Kowal P, Hestekin H, O’Driscoll T, Peltzer K, Yawson A, Biritwum R, Maximova T, Rodríguez AS, Espinoza BM, Wu F. Prevalence, risk factors and disability associated with fall-related injury in older adults in low-and middle-incomecountries: results from the WHO Study on global AGEing and adult health (SAGE). BMC Med. 2015 Dec;13(1):147.

8. World Health Organization, World Health Organization. Ageing, Life Course Unit. WHO global report on falls prevention in older age. World Health Organization; 2008.

9. Mitchell-Fearon K, James K, Waldron N, Holder-Nevins D, Willie-Tyndale D, Laws H, Eldemire-Shearer D. Falls among communitydwelling older adults in Jamaica. SAGE Open. 2014 Dec 20;4(4):2158244014564351.

10. Campbell B. Patient falls: Preventable, not inevitable. Nursing made Incredibly Easy. 2016 Jan 1;14(1):14 - 8.

11. Shojania KG, Marang-van de Mheen PJ. Temporal trends in patient safety in the Netherlands: reductions in preventable adverse events or the end of adverse events as a useful metric?. 
12. Cangany M, Back D, Hamilton-Kelly T, Altman M, Lacey S. Bedside nurses leading the way for falls prevention: An evidence-based approach. Critical care nurse. 2015 Apr;35(2):82-4.

13. Seo OI. Influence of knowledge and attitude on behaviors of falls prevention activities in patient among university hospital nurse. Unpublished master's thesis, The Catholic University of Korea, Seoul. 2008.

14. Kim CG. Nurses' knowledge and attitude toward fall in hospitalized patients. Journal of Industrial Science Researches.

2011;28(2):275-81.

15. Kim MH, Jeon HW, Chon MY. Study on the knowledge and attitudes of falls and awareness of fall risk factors among nursing students. Indian Journal of Science and Technology. 2015 Jan 1;8:74.

16. Laing SS, Silver IF, York S, Phelan EA. Fall prevention knowledge, attitude, and practices of community stakeholders and older adults. Journal of aging research. 2011;2011.

17. Choi BH. The nursing student's attitude and preventive activities on inpatients falls [Unpublished master's thesis]. Daegu: Keimyung University; 2011.

18. Asiri F, ALMohiza MA, Faia Aseeri M, Mehtab Alam M, Ataalla SM, Alqahtani M, Alshahrani A. Fall prevention knowledge and practice patterns among home healthcare professionals in southern Saudi Arabia: an observational study. Journal of international medical research. 2018 Dec;46(12):5062-73.

19. Gutta S, Joseph A, Chakraborty A, Alexander AM. Study on the knowledge, attitudes and practices regarding prevention of recurrent falls in the elderly. IOSR Journal of Dental Medical Sciences. 2013;9(3):32-8.

20. Kalu ME, Vlachantoni A, Norman KE. Knowledge about risk factors for falls and practice about fall prevention in older adults among physiotherapists in Nigeria. Physiotherapy research international. 2019 Jan;24(1):e1742.

\section{Figures}

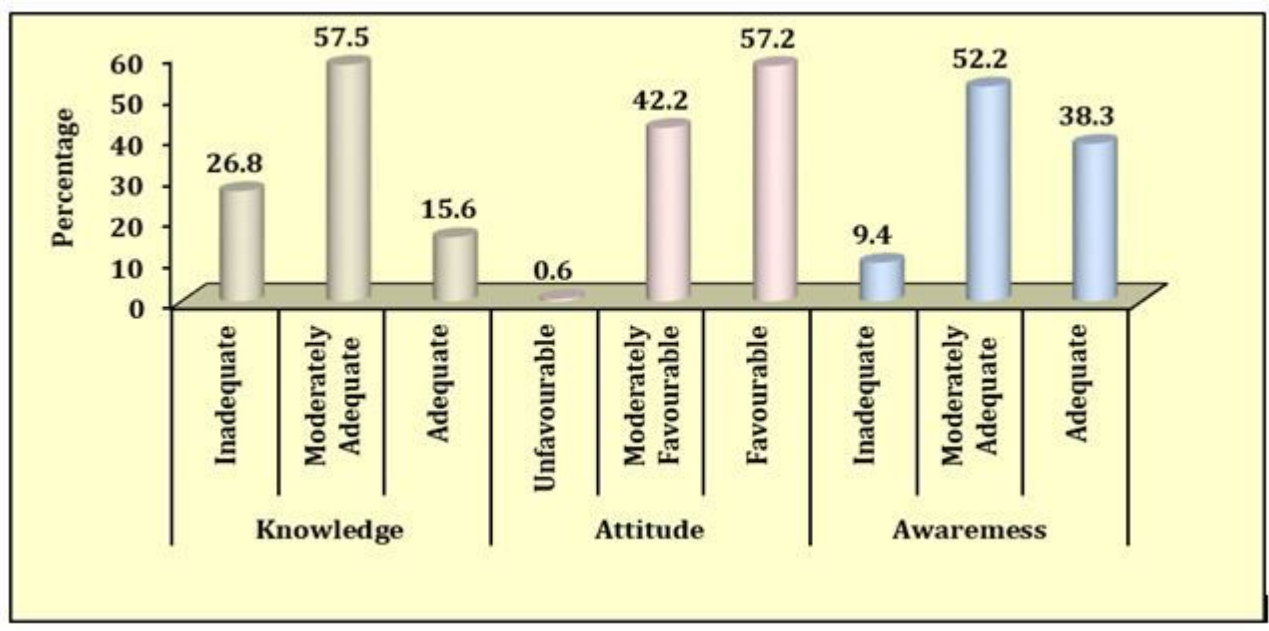

\section{Figure 1}

Overall percentage distribution of level of knowledge \& attitude on fall and awareness of fall risk factors. 